\title{
DEFENSA DE LA EPISTEMOLOGÍA ANTI-SUERTE: EN TORNO A LA RELACIÓN ENTRE SEGURIDAD Y HABILIDAD
}

\author{
Abel Wajnerman Paz \\ abelwajnerman@gmail.com \\ Universidad de Buenos Aires, CONICET, CLP
}

\begin{abstract}
Resumen / Abstract
Pritchard (2012) propone revitalizar el proyecto analítico para la noción de conocimiento: ofrecer una caracterización informativa y no circular de la misma. Cree que la clave es entender que las dos intuiciones centrales a esta noción imponen demandas independientes. Sostengo, por el contrario, la suficiencia de la condición anti-suerte. No solo respondo al argumento de Pritchard sino que desarrollo una línea alternativa inadvertida por él, pero implicada por supuestos suyos sobre la condición anti-suerte. Sostengo que una concepción evidencial de la seguridad la hace dependiente de una condición de habilidad. Fortalezco la defensa de la epistemología anti-suerte minando dicha concepción.

Palabras clave: conocimiento, condición anti-suerte, seguridad, problema de la generalidad, evidencialismo.

A DEFENSE OF ANTI-LUCK EPISTEMOLOGY:

ON THE RELATION BETWEEN SAFETY AND ABILITY

Pritchard (2012) proposed to revitalize the analytic project about knowledge: offering an informative and non-circular characterization. He believes that this depends on noticing that two main intuitions about knowledge imply two independent conditions. I argue that we can characterize knowledge using only the anti-luck condition. I do not offer only a response to Pritchard's argument but I also develop an alternative strategy, unnoticed by Pritchard, but based on his own assumptions regarding the anti-luck condition. I claim that an evidentialist characterization of safety makes it dependent on an ability condition. I strengthen the defense of anti-luck epistemology discussing this characterization.
\end{abstract}

KEY WORDS: Knowledge, anti-luck condition, safety, generality problem, evidentialism.

\section{Introducción}

R⿴囗十 Desde hace un tiempo, la tarea de ofrecer una elucidación de la noción de conocimiento, una caracterización informativa, no circular y que pueda acomodar nuestras intuiciones epistemológicas más salientes, ha caído en descrédito dados los numerosos intentos fallidos de llevarla a cabo. Recientemente, Pritchard (2012a) se ha propuesto superar este escepticismo respecto de lo que llama "el proyecto analítico" en teoría del conocimiento. Su propuesta parte de un diagnóstico peculiar respecto del 
fracaso del proyecto analítico. Considera que las dificultades principales derivan de no haber distinguido claramente dos intuiciones sobre el conocimiento.

La primera es la intuición familiar de que cuando uno conoce, el éxito cognitivo (que uno tenga una creencia verdadera) no se da por suerte. Siguiendo a Pritchard, podemos llamar a esta intuición la "intuición anti-suerte". Como señala Pritchard, esta intuición tiene un papel destacado en la teoría contemporánea del conocimiento. La respuesta estándar a por qué una creencia verdadera puede no bastar para el conocimiento es que la creencia puede ser verdadera por suerte ${ }^{1}$. A su vez, se afirma usualmente que lo que los casos Gettier muestran es que la creencia verdadera justificada puede no ser conocimiento porque en muchos casos ninguno de estos tres factores excluye la suerte ${ }^{2}$.

La segunda intuición, que Pritchard llama "la intuición de habilidad", es la de que el conocimiento requiere habilidad cognitiva en el sentido de que cuando uno sabe, el éxito cognitivo se debe a la habilidad cognitiva del sujeto. Este es otro presupuesto que figura en muchas consideraciones introductorias sobre la diferencia entre creencia verdadera y conocimiento ${ }^{3}$. Se considera de modo estándar que el problema con una creencia verdadera que no constituye conocimiento puede ser que no haya sido formada del modo correcto, mediante un proceso que sea adecuado para la adquisición de conocimiento. Parece claro, según Pritchard, que un proceso adecuado para la adquisición de conocimiento no es otra cosa que una habilidad cognitiva ${ }^{4}$.

Comúnmente se considera que estas intuiciones están vinculadas conceptualmente, que un compromiso con una implica un compromiso con otra. Frecuentemente el éxito cognitivo no se da por suerte si es el resultado de una habilidad cognitiva y, conversamente, si el éxito cognitivo no se da por suerte, entonces el resultado del ejercicio de una habilidad cognitiva. Si esto es así, si estas dos intuiciones son dos caras de una misma intuición, entonces uno esperaría que al formular adecuadamente una condición epistémica para excluir la suerte (y que podemos llamar con Pritchard "la condición anti-suerte"), esta nos permitirá dar cuenta a su vez de la intuición de habilidad (al menos en el sentido de que no implicará que hay casos de conocimiento en los que el sujeto no ejercita una habilidad cognitiva). Por otro lado, si formulamos adecuadamente una condición epistémica basada en la intuición de habilidad, una "condición de habilidad", esta nos permitirá dar cuenta también de la intuición antisuerte (al menos en el sentido de que no implicará que hay casos de conocimiento en los que la creencia es verdadera por suerte $)^{5}$.

Pritchard (2012a) argumenta que la tesis sobre la relación entre estas dos intuiciones es falsa. Considera que estas imponen demandas epistémicas independientes sobre una teoría del conocimiento y que solo cuando uno reconoce este hecho puede

Ver, por ejemplo, Steup (2006), sección 1.2.

Ver, por ejemplo, Dancy (1985), 134.

Ver, por ejemplo, Truncelitto 2007, sección 2.c.

Pritchard (2012a), pp. 247 y 248.

Pritchard (2012a), pp. 248 y 249. 
ofrecer una elucidación adecuada de la noción de conocimiento. Para argumentar a favor de este punto explora dos populares enfoques contemporáneos, cada uno de los cuales se centra en una de las dos intuiciones mencionadas. El primer enfoque, que Pritchard llama "epistemología anti-suerte" toma como medular la intuición anti-suerte e intenta ofrecer una caracterización del conocimiento que pueda dar cuenta de ella, pero que implique también dar cuenta de la intuición de habilidad. La segunda, conocida como "epistemología de la virtud", toma la intuición de habilidad como su núcleo e intenta ofrecer una caracterización del conocimiento que pueda dar cuenta de esta, pero que implique también dar cuenta de la intuición anti-suerte. Pritchard argumenta que ambas propuestas fallan, y lo hacen precisamente porque son incapaces de dar cuenta de la intuición que no toman como primordial. Para mostrar esto, Pritchard (2012a) propone en primer lugar un ejemplo, TEMP, en el que no hay conocimiento porque no se satisface la intuición de habilidad aunque sí la anti-suerte. En segundo lugar, propone una serie de ejemplos en los que no hay conocimiento porque no se satisface la intuición anti-suerte, aunque sí la de habilidad. Las fallas de estas propuestas pueden ser superadas, según Pritchard, si tomamos a las dos intuiciones como imponiendo demandas independientes, como requiriendo dos condiciones diferentes para el conocimiento. El resultado de satisfacer estas demandas es la posición que Pritchard llama "epistemología anti-suerte de la virtud" y que nos permitiría responder a los problemas que enfrentan los otros dos enfoques.

En este trabajo argumento, en contra de Pritchard, que las intuiciones están efectivamente relacionadas en el sentido que sugiere la epistemología anti-suerte. En particular, sostengo que no hay buenas razones para pensar que la satisfacción de una condición anti-suerte no implique la satisfacción de una condición de habilidad. En este sentido, una formulación adecuada de la condición anti-suerte sería suficiente para caracterizar el conocimiento. La peculiaridad de la defensa que ofrezco aquí reside en que no solo respondo al argumento propuesto por Pritchard sino que, sobre la base de los problemas que señalo a esta línea argumentativa, desarrollo una línea alternativa inadvertida por Pritchard, pero que se sigue de ciertos supuestos suyos sobre la condición anti-suerte. Proponiendo una respuesta a esta estrategia considero que ofrezco una defensa más sólida de la epistemología anti-suerte.

En la sección 2 considero el caso TEMP, que Pritchard (2012a) propone en contra de la epistemología anti-suerte. Argumento que el caso no funciona porque a pesar de que el proceso relevante tiene características muy atípicas, son características compartidas con casos en los que sí hay habilidad. Por este motivo, considero que en lugar de centrarse en casos atípicos que no son claramente incompatibles con habilidades cognitivas, un caso más sólido en contra de la epistemología anti-suerte debe mostrar que la condición anti-suerte puede satisfacerse aun cuando no se de alguna característica general que los epistemólogos de la virtud consideren explícitamente constitutiva de una habilidad. En las secciones que siguen muestro que una estrategia de este tipo está disponible si aceptamos ciertos supuestos de Pritchard sobre la condición anti-suerte. En particular, considero que dos supuestos de Pritchard sobre esta condición implican que una creencia puede satisfacer la condición anti-suerte aún cuando la facultad que la produzca no sea confiable y por lo tanto no constituya una habilidad. 
En la sección 3 me ocupo de explicitar el primer supuesto relevante. Pritchard (2012a) considera que la condición anti-suerte es relativa a la base que forma nuestra creencia. Sostengo que dado el problema de la generalidad para las propuestas confiabilistas, debemos encontrar un modo general de individuar esta base, aplicable a cualquier caso. En la sección 4 hago explícito el segundo supuesto. Señalo que el compromiso de Pritchard (2015) con la idea de que la suerte evidencial no es un tipo de suerte epistémica implica un compromiso con que la base para la condición antisuerte tiene que ser evidencial, es decir, que tiene que identificarse con una evidencia específica $e$ actualmente empleada. Ahora bien, sostengo que si adoptamos este criterio para individuar la base podemos revitalizar el argumento en contra de la epistemología anti-suerte. Pues el hecho de que una habilidad cognitiva puede emplear diferentes representaciones para producir una misma creencia, abre la posibilidad conceptual de que una facultad no sea confiable (y por lo tanto no permita satisfacer la condición de habilidad) aunque la evidencia actual que toma como input sí lo sea (y por lo tanto permita satisfacer la condición anti-suerte). La caracterización evidencial de la base de la condición anti-suerte permite un desfasaje entre esta y la de habilidad.

En la sección 5, argumento que un criterio evidencial para individuar la base de la condición anti-suerte no es sostenible. En particular, tomo de Pritchard mismo (Pritchard 2012b) un tipo de caso sobre el que el criterio evidencial nos arroja un resultado inadecuado. Propongo que la individuación en base a funciones psicológicas, propuesto por Alston (1995) funciona adecuadamente para explicar estos casos. Concluyo que si adoptamos esta propuesta para individuar la base de la condición anti-suerte, entonces ya no se da el desfase entre las dos condiciones. Por lo tanto, no tenemos razones para pensar que la epistemología anti-suerte no pueda caracterizar adecuadamente al conocimiento.

\section{TEMP y habilidades atipicas}

Antes de pasar a considerar el caso que propone Pritchard en contra de la epistemología anti-suerte, es importante aclarar un punto respecto de la intuición anti-suerte. Pritchard (2012a) defiende una versión particular de la condición anti-suerte: la condición de seguridad. Considera que la seguridad permite lidiar mejor que su rival más saliente, la condición de sensibilidad, con una serie de casos clave de suerte epistémica ${ }^{6}$. Más recientemente, Pritchard (2015) propone una defensa peculiar de la condición de seguridad basándose no en la consideración de casos específicamente epistémicos sino en una noción modal de suerte en general ${ }^{7}$. Brevemente, una creencia es segura si y solo si la base que la produce en el mundo actual no produce creencias falsas en mundos

Pritchard (2012a), pp. 249-259.

La defensa más acabada de su concepción modal de la suerte se encuentra en Pritchard (2014). 
cercanos $^{8}$. En lo que resta del trabajo no discutiré esta caracterización de la noción anti-suerte sino que, dándola por sentado, consideraré la tesis de Pritchard entendida ahora como la tesis de que la satisfacción de la condición de seguridad no implica ni es implicada por la satisfacción de la condición de habilidad. Como adelanté en la introducción, en el resto del trabajo argumento que no tenemos buenas razones para pensar que al menos seguridad implica habilidad.

Pritchard (2012a) propone un caso en el que se satisface la condición de seguridad, pero esto presuntamente no se debe al funcionamiento de una habilidad cognitiva. El caso TEMP consiste en un sujeto, Temp, que forma sus creencias sobre la temperatura de un cuarto consultando un termómetro. Sus creencias son seguras en tanto cualquier creencia formada sobre esta base será verdadera en situaciones actuales y contrafácticas cercanas. Más aún, Temp no tiene razón alguna para pensar que algo funciona mal con el termómetro. Pero ocurre que el termómetro está en realidad roto y que fluctúa arbitrariamente dentro de un rango. Sin que Temp lo sepa, hay un agente escondido en el cuarto que controla el termostato y cuyo trabajo es asegurarse de que cada vez que Temp consulte el termómetro, la temperatura que éste indique se corresponda con la temperatura en el cuarto.

Intuitivamente, según Pritchard, Temp no puede saber la temperatura del cuarto incluso si tenemos la garantía de que sus creencias serán verdaderas, es decir, incluso si sus creencias son seguras. El problema es que aparentemente el éxito cognitivo de Temp (que su creencia sea verdadera) no es causado por su habilidad cognitiva. La razón central por la que, según Pritchard, la condición de seguridad permite que una creencia segura no sea adquirida por medio de una habilidad es que las condiciones modales solo aseguran que haya una correspondencia entre hecho y creencia a lo largo de un conjunto de mundos relevantes. Pero no implican el tipo de relación específica requerida para que esa correspondencia sea el resultado de una habilidad cognitiva9 .

Ahora bien, la creencia de Temp sí es causada por cierto proceso cognitivo. En particular, Temp adquiere creencias sobre la temperatura a partir de representaciones perceptuales de las diferentes lecturas del termómetro. Hay un proceso cognitivo mediando la adquisición de su creencia verdadera. Podemos preguntarnos en qué sentido este proceso no implica el tipo de relación entre hechos y creencias que presupone el ejercicio de una habilidad cognitiva. Argumento que las tres respuestas sugeridas por Pritchard deben ser descartadas en tanto refieren a factores que, si bien son características irregulares o no usuales de procesos cognitivos, son compartidos por casos en los que sí atribuimos habilidad cognitiva. De este modo, no parece haber buenas razones para afirmar que no se cumple la condición de habilidad en TEMP.

En primer lugar, Pritchard afirma que el hecho de que la creencia de Temp sea verdadera depende muy fuertemente de factores del entorno externo ${ }^{10}$. Ahora bien, la 
dependencia respecto de factores externos al sujeto es algo que se aplica a cualquier habilidad cognitiva que sí nos permite adquirir conocimiento. Pritchard y Kallestrup (2014) mismos conceden a Greco (2009) que la manifestación de cualquier habilidad es relativa a ciertas condiciones del entorno ${ }^{11}$. Para ejercitar mis habilidades visuales, por ejemplo, hace falta que se den ciertas condiciones en el entorno (como iluminación, distancia, etc.) que hacen que esta produzca mayormente creencias verdaderas. De este modo, la mera dependencia de factores externos no puede impedir que podamos atribuir habilidad a Temp.

Tal vez el problema que ve Pritchard no es tanto que la habilidad de Temp dependa de condiciones externas sino el hecho de que lo externo sea un agente cognitivo diferente de Temp y que, de este modo, la verdad no depende de la agencia cognitiva de Temp mismo sino de alguien más ${ }^{12}$. Considero que Pritchard tampoco puede sostener que este sea un factor que impida la adquisición de conocimiento. La razón es que en muchos casos de conocimiento testimonial sucede precisamente esto: adquirimos conocimiento aun cuando la verdad de nuestra creencia depende mayormente de los procesos cognitivos de otro sujeto (en este caso, el que ofrece testimonio). Pritchard mismo considera que no debemos ser escépticos respecto del conocimiento testimonial. Más aún, los casos de conocimiento testimonial son una pieza clave en su argumentación en contra de la epistemología de la virtud.

En el caso JENNY, Jenny se baja de un tren en una ciudad desconocida y le pregunta cómo llegar caminando a su destino a un desconocido. Ocurre que la persona conoce bien la ciudad y le da direcciones correctas. Jenny cree lo que se le dice y sigue camino a su destino ${ }^{13}$. El caso es problemático para la epistemología de la virtud porque diríamos que Jenny tiene conocimiento a pesar de que no diríamos que su éxito cognitivo se debe primariamente al ejercicio de sus habilidades cognitivas (sino más bien a las de su interlocutor). Ahora bien, este caso parece prima facie problemático para Pritchard mismo, ya que parece implicar que la habilidad cognitiva no es necesaria para el conocimiento, es decir, parece poner en duda la intuición de habilidad como una de las dos intuiciones que debe moldear nuestra concepción del conocimiento. Sin embargo, Pritchard considera que lo que en realidad nos muestra el ejemplo es que la condición de habilidad debe ser moderada. Los epistemólogos de la virtud han ofrecido una versión robusta de la condición de habilidad para poder dar cuenta de casos como el de las ovejas de Chisholm (1977), sin tener que acudir a una condición anti-suerte, ajena a su enfoque. Esta condición afirma que no basta que el sujeto ejerza una habilidad cognitiva sino que el éxito cognitivo se debe dar en virtud del ejercicio dichas habilidades. Pritchard cree que los casos de conocimiento testimonial funcionan en contra de esta versión robusta. Sin embargo, si moderamos la condición de habilidad para dar cuenta de estos casos de conocimiento se sigue, a favor de Pritchard, que

11 Pritchard y Kallestrup (2014), pp. 339-342.

$12 \quad$ Pritchard (2012a) p. 261.

13 Pritchard (2012a), p. 269. 
debemos complementar la condición de habilidad con algo más (presumiblemente una condición anti-suerte), justamente para dar cuenta de los casos como el de Chisholm.

Supongamos que concedemos a Pritchard que la condición de habilidad no debe ser formulada de un modo robusto y que por lo tanto su satisfacción no implica la de la condición de seguridad. Esto quiere decir que en los casos de conocimiento testimonial se satisface la condición de habilidad, aun cuando la verdad de la creencia dependa mayormente de una agencia cognitiva ajena. Pero si este es el caso, la agencia cognitiva externa tampoco implica la ausencia de habilidad en Temp. El hecho de que la verdad de la creencia de Temp dependa mayormente de un agente externo no implica que Temp no satisfaga la condición de habilidad.

Por último, la impresión de que el éxito cognitivo no depende de la habilidad de Temp puede surgir, como afirma Pritchard, de que“se invierte la relación de adecuación". Esta expresión parece aludir al hecho de que se invierte la dirección causal entre lo que Temp toma como evidencia, las lecturas del termómetro, y los hechos que indican esa evidencia, la temperatura del ambiente. En los contextos usuales, las diferentes temperaturas causan diferentes lecturas en un termómetro, pero en TEMP las diferentes lecturas causan, por medio del agente oculto, diferentes temperaturas. Nuevamente, no creo que esta inversión implique una irregularidad problemática para el proceso cognitivo de Temp. En muchos casos, la evidencia respecto de un hecho $p$ es la causa y no el efecto de $p$. Comúnmente podemos rastrear una propiedad no solo por medio de sus efectos sino también por medio de sus causas. Por ejemplo, podemos rastrear confiablemente instancias de la propiedad de estar hirviendo de una muestra de agua $a$, tomando como evidencia información sobre la temperatura de $a$, la causa de la instanciación de dicha propiedad. Por este motivo, la inversión causal entre hechos y evidencia no implica que Temp no cumpla una condición de habilidad. Como las diferentes características de TEMP que Pritchard señala como indicativas de ausencia de habilidad son compartidas por casos de habilidad, considero que no tenemos fundamento genuino para afirmar, a partir de este caso, que puede haber seguridad sin habilidad. TEMP explota diferentes características atípicas del proceso empleado para argumentar a favor de la ausencia de habilidad. Dada la variedad de procesos cognitivos de los que disponemos, como vimos, es plausible que encontremos estas características en procesos que sí consideramos que constituyen la manifestación de una habilidad. Considero por este motivo que una estrategia más sólida podría consistir en mostrar que la seguridad puede darse aun cuando no se dé alguna de las características que los epistemólogos de la virtud consideran explícitamente constitutivas de una habilidad cognitiva. En las próximas dos secciones intentaré desarrollar una estrategia de este tipo a partir de ciertos supuesto de Pritchard sobre la condición de seguridad.

\section{Relatividad a base y generalidad}

En la sección siguiente argumentaré que si concedemos a Pritchard ciertos supuestos generales sobre la condición de seguridad se sigue (inadvertidamente para él) una razón prima facie más sólida en contra de la epistemología anti-suerte. Pues esta noción de 
seguridad abre la posibilidad conceptual de que se cumpla la condición anti-suerte sin que se dé cierta característica que muchos epistemólogos de la virtud aceptarían como necesaria para la instanciación de una habilidad: la confiabilidad. Como señala Pritchard (2012a) es generalmente admitido que uno de los requisitos mínimos para que algo cuente como una habilidad es la confiabilidad ${ }^{14}$

En esta sección y en la siguiente mostraré cuál es la motivación para los supuestos relevantes que ponen en marcha esta estrategia. Hay dos supuestos de Pritchard que apuntan en esta dirección. En primer lugar, el supuesto de que para ofrecer una caracterización adecuada de la condición de seguridad, esta debe hacer referencia a la base a partir de la que formamos la creencia evaluada. En segundo lugar, considero que la tesis de Pritchard de que la suerte evidencial no mina el conocimiento implica un compromiso con la tesis de que la base de la seguridad debe ser entendida en un sentido evidencialista. Al final de la próxima sección, mostraré en qué sentido estos dos supuestos implican la posibilidad de que una creencia sea segura sin que la facultad que la produce sea confiable y, por lo tanto, sin que constituya una habilidad.

Empiezo por explicitar en esta sección la relación entre seguridad y base. Supongamos que decidimos caracterizar la seguridad del siguiente modo: Una creencia $\mathrm{C}$ en una proposición p por parte de un sujeto $\mathrm{S}$ es segura si y solo si $\mathrm{C}$ es verdadera en todos los mundos cercanos en los que es adquirida por S. Si construimos la seguridad de este modo, se sigue que cualquier creencia en una proposición necesaria (como las matemáticas) o incluso en una proposición no necesaria pero cuya verdad es modalmente estable (como las proposiciones sobre leyes físicas o incluso sobre regularidades no estrictas) será segura. Pues si la verdad de una proposición p es modalmente estable entonces no va a haber mundo posible cercano en el que el agente cree falsamente que p. Esto es un problema, porque claramente hay situaciones en las que diríamos que la verdad de una creencia de este tipo se da por suerte. Imaginemos, por ejemplo, que un sujeto forma una creencia verdadera en una proposición matemática, decide creer en ella, tirando una moneda. No hay mundo cercano en el que forme una creencia falsa en esa proposición, pero el hecho de que haya acertado, de que su creencia sea verdadera, es claramente una cuestión de suerte y eso explica que no tenga conocimiento.

La respuesta de Pritchard (2015) a este tipo de casos es sostener una caracterización de la seguridad relativa a cierta base. Una creencia es segura si y solo si el sujeto no creería fácilmente proposiciones falsas sobre la misma base. Las creencias matemáticas adquiridas sobre bases inadecuadas son inseguras porque, aunque ellas mismas no sean falsas en mundos cercanos, hay mundos cercanos en los que el sujeto adquiere creencias falsas sobre la misma base. En particular, hay mundos cercanos en los que el sujeto cree una proposición matemática falsa tirando una moneda ${ }^{15}$.

14 Pritchard (2012a), p. 262. Pritchard señala que esta caracterización estándar de las habilidades epistémicas se puede rastrear hasta Greco (1999) y Greco (2000) (por supuesto, no es la única caracterización disponible).

15 Pritchard (2015), p. 102 y 103. 
A pesar de que la restricción de relatividad a base está sólidamente motivada tiene, a mi parecer, un costo. Si se acepta, el defensor de la condición de seguridad debe ahora hacerse cargo de problemas propios de otras formas de confiabilismo que una noción menos restrictiva de la seguridad permitía eludir. En particular, considero que la noción de seguridad de Pritchard debe dar una respuesta al llamado "problema de generalidad".

Este problema para el confiabilismo ${ }^{16}$ ha sido sistemáticamente enfatizado por Conee y Feldman ${ }^{17}$. La idea es que una creencia particular es el producto de un proceso causal caso, un proceso concreto que ocurre en un tiempo y lugar precisos. Este proceso caso es una instancia de diferentes tipos, más amplios o más estrechos (más generales o más específicos). Cada tipo va a tener su propio nivel de confiabilidad, normalmente diferente de los niveles de confiabilidad de otros tipos. Por ejemplo, supongamos que, en un momento dado, formo una creencia a partir de un testimonio. Supongamos además que el proceso que causa esta creencia puede ser individuado de al menos dos formas diferentes: Podemos individuarlo como un caso del tipo de proceso de adquirir una creencia a partir del testimonio de $m i$ abogado o como un caso del tipo de proceso de adquirir una creencia a partir del testimonio de un abogado. Supongamos ahora que en la sociedad en la que me encuentro los abogados son generalmente poco confiables, pero que mi abogado es una excepción, que resulta altamente confiable. Si individúo al proceso por medio del primer tipo tendrá un grado mucho más alto de confiabilidad (o seguridad) que si lo hago por medio del segundo. La pregunta que surge entonces es: ¿Qué tipo de proceso debo elegir para asignarle al caso un grado determinado de confiabilidad?

Conee y Feldman (1998) proponen diferentes requisitos para una solución adecuada al problema de la generalidad. Estos han sido generalmente tomados como punto de partida por las diferentes propuestas de solución. El requisito que me interesa aquí es el de que la solución al problema debe darse por principio, en el sentido de que la especificación del tipo relevante que determina la confiabilidad del caso no debe ser arbitraria, no debe ser hecha ad hoc, caso por caso. Este requisito parece razonable, pues si no hubiese un criterio general para elegir el tipo relevante en cada caso que evaluamos epistémicamente, podremos siempre elegir el tipo que se acomoda mejor a nuestras intuiciones. Cuando estamos frente a un caso que intuitivamente es un caso de conocimiento podemos elegir el tipo que hace que se cumpla la condición confiabilista que queremos defender, y cuando estamos frente a un caso que intuitivamente no lo es, podemos elegir el que hace que no se cumpla. Esto haría a la forma de confiabilismo que nos interesa trivialmente verdadera.

Podemos preguntarnos ahora en qué sentido la seguridad relativa a la base nos lleva a enfrentarnos con el problema de la generalidad. El punto es el siguiente. Si la seguridad de una creencia $C$ de un sujeto $\mathrm{S}$ fuese meramente la verdad de $C$ en los

16 Ver Goldman (2008) para un repaso más pormenorizado de este problema.

17 Por ejemplo, Feldman y Conee (1985) y Conee y Feldman (1998). 
mundos cercanos en los que es adquirida por $\mathrm{S}$, entonces no tendríamos el problema de la generalidad. Pues como los mundos cercanos relevantes para la seguridad no serían relativos a un modo de adquisición (no serían solo los mundos en los que $\mathrm{S}$ adquiere $C$ del mismo modo que en el mundo actual), diferentes modos de individuar este modo de adquisición no delimitarían diferentes conjuntos de mundos cercanos relevantes para la seguridad (y por lo tanto, diferentes grados de seguridad).

Volvamos al caso del abogado mencionado anteriormente. Supongamos que adoptamos esta concepción de la seguridad no relativa a base, que sostenemos que la seguridad es solo la verdad de la creencia en los mundos cercanos en los que la adquiero de cualquier modo. Como según esta concepción los mundos cercanos en los que adquiero la creencia actual de un modo diferente al actual serán relevantes para evaluar la seguridad, mi creencia será insegura aun si individúo el modo actual de adquisición según el tipo adquirir una creencia sobre la base del testimonio de $\underline{\text { mi }}$ abogado. Pues aunque adquirir la misma creencia por medio del testimonio de abogados deshonestos, por ejemplo, constituya un modo diferente del actual, los mundos en los que empleo este modo alternativo serán también relevantes para la evaluación epistémica. Diferentes modos de individuar la base no harían una diferencia respecto de la seguridad o confiabilidad.

Como vimos, la noción de seguridad motivada por el ejemplo de las proposiciones matemáticas y la noción general de suerte pierde esta característica. Debemos tener en cuenta el modo de adquisición de la creencia considerada para determinar los mundos posibles relevantes. De esta manera, como la base o modo de adquisición puede individuarse de diferentes maneras o instanciar diferentes tipos que, como vimos, determinan diferentes grados de confiabilidad, debemos ofrecer un criterio para individuar el modo de adquisición o elegir el tipo relevante.

Considero que la respuesta al problema de la generalidad que podemos atribuir a Pritchard es implicada por su consideración de lo que llama "suerte evidencial". En particular, mostraré en la próxima sección que supone un compromiso con una respuesta evidencialista al problema de la generalidad. Argumentaré que este tipo de respuesta abre la puerta a ciertos contraejemplos para la epistemología anti-suerte.

\section{Seguridad evidencial y funciones psicológicas}

Se ha ofrecido una amplia gama de respuestas al problema de la generalidad. Alston (1995) ha propuesto una estrategia que ha sido seguida por algunas de ellas ${ }^{18}$. Más aún, Comesaña (2006) ha argumentado más fuertemente que, si una teoría del conocimiento es posible, sea confiabilista o no, algo como la estrategia de Alston tiene que funcionar. 
Empezaré por considerar la idea de Alston y algunos puntos centrales de la discusión que ha suscitado.

Alston sostiene que como hay una cuestión de hecho respecto de qué características de la vida mental de un sujeto influyen en la formación de una creencia y de qué modo lo hacen, entonces siempre podemos recurrir a estas características para determinar el tipo de proceso relevante que determine el grado de confiabilidad de un proceso caso. En particular, Alston considera que toda formación de creencia involucra la instanciación o realización de una función psicológica. Esta función determina qué características del input causan la creencia output y de qué modo lo hacen. Así, cuando tenemos que evaluar la confiabilidad del proceso que produce una creencia, el tipo relevante va a estar determinado por la función responsable de la producción de dicha creencia ${ }^{19}$.

En las discusiones en torno a este tipo de respuesta se ha asumido frecuentemente que la función psicológica en cuestión puede caracterizarse como un mapa complejo de input-output. Este mapa describe cómo se vinculan las diferentes representaciones que la función toma como input ${ }^{20}$. Ahora bien, Comesaña $(2006,2010)$ ofrece una solución al problema de la generalidad que sigue también la línea de Alston (1995) pero que interpreta de un modo diferente su idea central. Argumenta que debemos emplear el tipo basar la creencia en $p$ en la evidencia e para determinar la confiabilidad de un proceso dado.

No me detendré aquí a considerar los detalles de la argumentación y la propuesta. Me interesa señalar solamente que este modo de individuar los procesos de adquisición difiere de la idea de tomar funciones psicológicas. Comesaña no toma a la función que mapea diferentes inputs con diferentes outputs sino solo al input actual relevante para la producción del output actual. El tipo basar la creencia en p en la evidencia e toma en consideración solo la representación actualmente tomada como input, la evidencia $e$ efectivamente empleada, y la representación específica que ese input produce como output (la creencia en $p$ ). Comesaña llama a su propuesta "confiabilismo evidencialista".

Considero que el compromiso de Pritchard (2015) con la tesis de que la suerte evidencial no constituye el tipo de suerte epistémica que puede minar el conocimiento implica un compromiso con un modo evidencialista de caracterizar la base de la seguridad. En particular, lo compromete con la idea de que una creencia adquirida a partir de una evidencia $e$ es segura si y solo si $e$ no produce creencias falsas en mundos cercanos.

Que la suerte evidencial es compatible con el conocimiento quiere decir que uno puede adquirir conocimiento a partir de cierta evidencia a pesar de que uno haya empleado esa evidencia por suerte (es decir, aunque emplee evidencia diferente en mundos cercanos). Supongamos que la evidencia actual no produciría creencias falsas en mundos cercanos. Si ocurre esto, la creencia es segura. En este caso, según Pritchard,

\footnotetext{
19 Alston (1995) p. 15.

20 Ver por ejemplo, Adler y Levin (2002), Conee y Feldman (1998), Feldman, and Conee (1985) y Feldman y Conee (2002).
} 
uno puede tener conocimiento a pesar de que la creencia actual es falsa en un mundo cercano en el que es producida por otra evidencia. En este sentido considero que Pritchard sostiene una caracterización evidencial de la seguridad.

Ahora bien, creo que la motivación que propone Pritchard para una noción evidencialista de la seguridad no es sólida. La razón que ofrece es que la seguridad es una noción relativa a cierta base ${ }^{21}$. Pritchard simplemente asume que la base debe identificarse con una evidencia específica. Como vimos, dado el problema de la generalidad, no es posible asumir esto. Tenemos que ofrecer alguna razón para identificar la base con la evidencia actual y no, por ejemplo, con el mapa complejo de input-output que toma esa evidencia como uno de sus inputs. Si elegimos este último modo de individuar la base, casos de suerte evidencial sí podrían contar como casos de suerte epistémica que impiden la adquisición de conocimiento.

Supongamos que identificamos la base $b$ sobre la que adquiero mi creencia $\mathrm{C}$ en una proposición $p$ con una función $F$ que toma diferentes representaciones como input $r_{1}$ y $r_{2}$ y arroja como output mi creencia en $p$. Supongamos, por ejemplo, que tengo una función que toma como input una de dos representaciones perceptuales, la representación auditiva de un ladrido o la representación visual de la apariencia de un perro, y arroja como output la creencia de que hay un perro en el entorno. En un momento dado, oigo un ladrido y adquiero la creencia de que hay un perro en mi entorno. Como mi audición funciona adecuadamente, no hay mundos cercanos en los que adquiera la misma creencia sobre dicha representación auditiva y la creencia sea falsa. Sin embargo, como mi visión es defectuosa, habrá mundos cercanos en los que la misma función toma la representación visual como input y produzca la creencia falsa de que hay un perro en el entorno. Según si identifico la base de mi creencia con la función psicológica empleada actualmente o con la evidencia actual surgirán diferentes veredictos respecto de la seguridad de mi creencia.

En la próxima sección argumentaré en contra de la lectura evidencial de la seguridad y a favor de identificar a la base con funciones psicológicas. Pero antes de llegar a ese punto quisiera mostrar de qué manera considero que la lectura evidencial de la seguridad puede ser problemática para la epistemología anti-suerte. Si esta implicación es correcta, mi rechazo de la lectura evidencial en la próxima sección equivale a una defensa de la epistemología anti-suerte.

El punto se sigue de un modo bastante directo a partir de lo ya dicho. Las habilidades cognitivas se asemejan a las funciones psicológicas en que pueden tomar una variedad de representaciones como input y producir una variedad de representaciones como output. Por ejemplo, la percepción visual, que los epistemólogos de la virtud toman como paradigma de habilidad cognitiva, puede tomar una amplia variedad de representaciones como input, cualquiera de las experiencias visuales de las que nuestra facultad visual es capaz, y producir como output una variedad de creencias perceptuales. $\mathrm{Si}$, adoptando una perspectiva evidencialista, identificamos a la base para la seguridad 
con la evidencia actualmente empleada, entonces se sigue inmediatamente que una creencia puede ser segura aunque la facultad que la produce no sea confiable y por lo tanto no constituya una habilidad. Pues aunque la evidencia que produce la creencia no produzca creencias falsas en mundos cercanos (y por lo tanto haga segura a la creencia), la facultad que toma esa evidencia como input puede producir creencias falsas en mundos cercanos en los que toma inputs diferentes al actual y por lo tanto ser no confiable. De este modo, la noción evidencialista de seguridad implica un desfase posible entre seguridad y habilidad que vuelve a poner en duda a la epistemología anti-suerte.

\section{Seguridad cognitiva}

Considero que la noción evidencialista de seguridad no es sostenible y que el criterio más razonable no es problemático para la epistemología anti-suerte. El problema puede ilustrarse mediante la consideración de un caso que propone Pritchard (2012b) mismo ${ }^{22}$. Considero que si Pritchard está en lo cierto y el caso que propone no es un caso de conocimiento, entonces se sigue que no podemos adoptar el criterio de Comesaña para individuar funciones psicológicas.

El caso de Pritchard, llamado "CAR", es una adaptación de un caso clásico ofrecido por Goldman (1976) de las fachadas de granero y aparentemente desafía a la seguridad como condición necesaria para el conocimiento, es decir, es aparentemente un caso de creencia insegura que constituye conocimiento. Pritchard argumenta (y yo lo seguiré en esto) que el caso describe una creencia que no es segura pero tampoco constituye conocimiento.

CAR consiste en un niño, Alexander, que es muy bueno para reconocer el tipo de auto específico que su padre maneja, que es un Vauxhall Zafira. En el entorno en el que está, Alexander no confundiría fácilmente un Vauxhall Zafira con otro tipo de vehículo. En general, sin embargo, Alexander no es bueno para identificar autos en su entorno. Clasificaría la mayoría de los vehículos que ve como autos, incluyendo camiones, autobuses, etc. Como hay muchos vehículos en su entorno que Alexander clasificaría falsamente como autos, clasificaría falsamente un vehículo como auto muy fácilmente. El problema que este caso presenta es el siguiente. Supongamos que Alexander ve un Vauxhall Zafira estacionado frente a su casa y forma la creencia de que hay un auto estacionado frente a su casa. Como Alexander tiene una tendencia a clasificar erróneamente vehículos en su entorno como autos, parece haber mundos posibles cercanos en los que forma su creencia del mismo modo que en el mundo actual (es decir, percibiendo visualmente el frente de su casa) y cree falsamente que hay un auto estacionado (por ejemplo, cuando hay un camión estacionado frente a su casa). Si consideramos que este caso constituye un caso de conocimiento, tendríamos un caso de conocimiento no seguro, lo que atentaría contra la seguridad como condición necesaria. 
Pritchard ofrece razones para pensar que en este caso no hay conocimiento y aquí coincidiré con él. Pero antes de considerar estas razones es importante enfatizar un punto. Este caso es problemático para Pritchard porque considera que efectivamente la creencia de Alexander es insegura. Pero la creencia es inseguro solo si no identificamos a la base para determinar la seguridad con la evidencia efectivamente empleada en la situación actual (el input actual del proceso de formación de su creencia). Pues la situación alternativa que mina la seguridad de la creencia de Alexander es tal que la evidencia empleada (la experiencia de ver un camión) es diferente de la evidencia empleada en la situación actual (la experiencia de ver un Vauxhall Zafira). Las consideraciones de Pritchard sobre este caso, que describiré brevemente a continuación, determinan que no solo la seguridad, sino también el conocimiento requiere que la creencia sea verdadera en situaciones contrafácticas de este tipo.

Pritchard nos pide que consideremos que somos uno de los padres de Alexander y que tenemos un invitado que está esperando que llegue un auto a buscarlo. Consideremos estos dos escenarios. En el primer escenario, Alexander entra al cuarto y le dice al invitado que hay un auto estacionado afuera. En este caso, uno le advertiría al invitado que Alexander tiende a clasificar todo tipos de cosas que no son autos como autos y que por lo tanto debería tener cuidado en formar su creencia de que hay un auto sobre la base del testimonio de Alexander. Pero esto parece implicar que Alexander no tiene conocimiento de esta proposición. Más aún, lo mismo diríamos del invitado si, no habiéndole advertido sobre Alexander, forma su creencia sobre la base del testimonio. Pues si Alexander supiese que hay un auto afuera, entonces parece que el invitado debería ser capaz de adquirir conocimiento sobre la base de su testimonio.

Si Pritchard está en lo correcto, podemos extraer la siguiente conclusión relevante para la evaluación de la caracterización evidencialista de la seguridad. Aun cuando una creencia no sea falsa en los mundos cercanos en los que es producida por la misma evidencia, diríamos que no es segura, y que por lo tanto no es conocimiento si es falsa en mundos cercanos en los que es adquirida por medio de otra evidencia. Si la creencia hubiese sido fácilmente adquirida por medio de otra evidencia en una situación en la que es falsa, entonces es insegura. Esto quiere decir que la base que necesitamos para determinar la condición de seguridad no puede ser la que surge de la propuesta evidencialista, no puede ser la evidencia específica efectivamente empleada.

Por el contrario, si tomamos el mapa complejo de input-output de la función efectivamente empleada por Alexander, siguiendo la versión original de la propuesta de Alston, entonces se sigue un veredicto adecuado sobre este tipo de casos. Para determinar cuál es el mapa de input-output que define la función empleada hay que determinar primero qué tipo de tarea cognitiva está llevando a cabo el sujeto. La tarea cognitiva de Alexander es la tarea experimental de identificación de categoría, de determinar si un objeto dado cae bajo una categoría dada. En este caso, debe determinar si el objeto percibido es un auto. Prinz (2002) es uno de los filósofos de las ciencias cognitivas que se ha ocupado de dar una caracterización pormenorizada de este tipo de mecanismos. Siguiendo a Prinz, podemos afirmar que los mecanismos instanciados en la tarea de identificación de miembros de una categoría dada son los mecanismos de detección que conforman la red de memoria de esa categoría. 
Veamos brevemente en qué consisten estas redes. Prinz (2002) propone una teoría de conceptos peculiar. Identifica a los conceptos con lo que llama "proxytipos". Los proxytipos son representaciones perceptuales que podemos emplear para detectar a los miembros de una categoría dada y que a su vez pueden desempeñar, según Prinz, todas las funciones que se espera que cumplan los conceptos. Para la tarea epistémica presente, permanezco neutral respecto de estas tesis más sustantivas. Solo me interesa el hecho de que, para una categoría dada, contamos con una red de representaciones en la memoria a largo plazo (los proxytipos de esa categoría), constituida por representaciones de ejemplares, representaciones prototípicas o representaciones de propiedades más ocultas o teóricas (causales, por ejemplo) de dicha categoría. Cualquiera de estas representaciones puede funcionar, en diferentes ocasiones, para detectar miembros de dicha categoría ${ }^{23}$.

Se sigue de lo dicho que la red de memoria, en tanto función o mecanismo de detección de miembros de una categoría, puede tomar como inputs diferentes representaciones de los miembros de dicha categoría. En particular tomará, entre otras, las representaciones de lo que el sujeto considera ejemplares de dicha categoría. Como Alexander tomará a ciertos camiones, al igual que a los Vauxhall Zafiras, por ejemplares de autos, las representaciones de ciertos camiones formarán parte del mecanismo de detección de autos de Alexander. Si identificamos la base de la creencia de Alexander con este mecanismo, entonces su seguridad no dependerá solo de las situaciones contrafácticas en las que cree que hay un auto sobre la base de la percepción de un Vauxhall Zafira sino también aquellas en las que lo cree sobre la base de la percepción de un camión. Esto implica que la creencia será insegura y que por lo tanto no constituirá conocimiento, como afirma Pritchard. De este modo, caracterizar la base de la seguridad en base a los mapas complejos de input-output que constituyen una función psicológica nos permite lidiar con CAR de un modo más adecuado que el enfoque evidencial.

Pero lo que más nos interesa es que, a favor de la epistemología anti-suerte, esta concepción de la seguridad nos permite bloquear la posibilidad de desfase entre habilidad y seguridad que había abierto la concepción evidencialista. Vimos que identificar la base de la seguridad con una evidencia $e$ específica permitía que haya seguridad sin habilidad porque abría la posibilidad de que una creencia sea verdadera en todos los mundos cercanos en los que es producida a partir de $e$ pero que la función que toma a $e$ como input no sea confiable porque produce creencias falsas en los mundos cercanos en los que toma otra evidencia $e_{1}$ como input. Pero si ahora identificamos a la base de la creencia con el conjunto de inputs que puede tomar la función que produce la creencia, entonces el desfase ya no puede darse. Si la función actual produce una creencia falsa en la situación contrafáctica en la que toma a $e_{1}$ como input, entonces la creencia actual es insegura. 


\section{Conclusión}

He intentado ofrecer una defensa de la epistemología anti-suerte frente a la objeción de Pritchard (2012a) de que no puede dar cuenta de la intuición de habilidad. En primer lugar he desestimado el caso propuesto por Pritchard. Sostuve que los únicos factores a los que podemos adjudicar la supuesta falla de Temp en satisfacer una condición de habilidad son factores comunes a casos donde diríamos que dicha condición se satisface. Casos en los que el éxito cognitivo de un sujeto se debe a su habilidad cognitiva pueden ser casos en los que la habilidad dependa de factores del entorno externo, en los que gran parte de la habilidad deba ser atribuida a otro agente cognitivo o incluso donde la relación causal entre evidencia y creencia es invertida.

En segundo lugar, he desarrollado y bloqueado una línea alternativa de argumentación que no presenta el problema del ejemplo de Pritchard. Sostuve que si aceptamos ciertos supuestos de Pritchard sobre la condición de seguridad se sigue que esta puede satisfacerse aun cuando no se dé un factor que cualquier epistemólogo de la virtud aceptaría como necesario para la manifestación de una habilidad: la confiabilidad.

Mostré que la relatividad a base de la noción de seguridad nos obliga a lidiar con el problema de la generalidad, a ofrecer un criterio general para individuar dicha base. Sostuve que el compromiso de Pritchard con la tesis de que la suerte evidencial no mina el conocimiento lo compromete con un criterio evidencialista para individuar la base. Argumenté que este criterio puede revitalizar la tesis de Pritchard de que puede haber seguridad sin habilidad.

Sostuve que este criterio no es sostenible porque implica resultados anti-intuitivos respecto de cierto tipo de casos. Mostré que un caso que Pritchard (2012b) mismo ofrece nos sirve para determinar que el enfoque evidencialista falla. Argumenté que, por el contrario, un enfoque que identifique la base de la seguridad con una función psicológica nos permite lidiar con el caso problemático. A su vez, este criterio para individuar la base cierra la posibilidad de un desfase entre seguridad y habilidad.

De estos puntos concluyo que la epistemología anti-suerte constituye aun un buen candidato para llevar adelante el proyecto analítico respecto de la noción de conocimiento. Esto no quiere decir que este enfoque proponga una caracterización más adecuada que la epistemología de la virtud. Es plausible que, en contra de Pritchard, las dos intuiciones sean efectivamente dos caras de una misma moneda. Pero para sostener esto habría que responder también al menos a los argumentos de Pritchard a favor de que puede haber habilidad sin seguridad. Los argumentos en este trabajo alcanzan para sostener que no tenemos buenas razones para pensar que puede haber seguridad sin habilidad. 


\section{Referencias bibliográficas}

Adler, J. and M. Levin (2002), "Is the Generality Problem too General?”, Philosophy and phenomenological Research LXV: 87-97.

Alston, W. (1995), "How to Think About Reliability", Philosophical Topics 23: 1-29

Beebe, J. (2004), “The Generality Problem, Statistical Relevance and the Tri-Level Hypothesis", Noûs 38: 177-195.

Comesaña, J. (2006), “A Well-Founded Solution to the Generality Problem”, Philosophical Studies 129: 27-47.

(2010), "EvidentialistReliabilism", Noûs 44: 571-600.

Conee, E. and R. Feldman (1998), "The Generality Problem for Reliabilism", Philosophical Studies 89: 1-29.

Feldman, R. and E. Conee (1985), "Evidentialism”, Philosophical Studies 48: 15-34. (2002), “Typing Problems", Philosophy and Phenomenological Research LXV: 98-105.

Goldman, A. (1976), 'Discrimination and Perceptual Knowledge', Journal of Philosophy 73: 771-91.

(2008), "Reliabilism",en E Zalta, ed., The Stanford Encyclopedia of Philosophy. URL: <http://plato.stanford.edu/archives/spr2011/entries/ reliabilism/>

Greco, J. (1999), “Agent Reliabilism,” Noûs, xxxiii, Supplement: Philosophical Perspectives 13, Epistemology: 273-96.

(2000), Putting Skeptics in Their Place: The Nature of Skeptical Arguments and Their Role in Philosophical Inquiry. New York, Cambridge.

Greco, J., \& J. Turri (2009), 'Virtue Epistemology', en E. Zalta, ed., Stanford Encyclopaedia of Philosophy. URL: http://plato.stanford.edu/entries/ epistemology-virtue/

Heller, M. (1995), "The Simple Solution to the Generality Problem”, Noûs 29: 501515.

Luper, S. (1984), “The Epistemic Predicament”, Australasian Journal of Philosophy 62: $26-50$.

(2003), "Indiscernability Skepticism", en S Luper, ed., The Skeptics: Contemporary Essays. Aldershot: Ashgate, 183-202.

Prinz, J. (2002), Furnishing the Mind: Concepts and Their Perceptual Basis. Cambridge: MA. MIT Press.

Pritchard, D., (2002), "Resurrecting the Moorean Response to the Sceptic", International Journal of Philosophical Studies 10: 283-307. 222.

(2004), "Epistemic Luck", Journal of Philosophical Research, 29: 193-

Pritchard, D. y M. Smith (2004), “The Psychology and Philosophy of Luck”, New Ideas in Psychology 22: 1-28. 
Pritchard, D. (2005), Epistemic Luck. Oxford: Oxford University Press. (2007), “Anti-Luck Epistemology”, Synthese 158: 277-97.

(2008), "Sensitivity, Safety, and Anti-Luck Epistemology", en J. Greco, ed., The Oxford Handbook of Scepticism. Oxford: Oxford University Press, 437-455. 247-279.

(2012a), “Anti-Luck Virtue Epistemology”, Journal of Philosophy 109:

(2012b), "In Defence of Modest Anti-Luck Epistemology", en T. Black y K. Becker, eds., The Sensitivity Principle in Epistemology. Cambridge: Cambridge University Pres, 173-192.

Pritchard, D. y J. Kallestrup (2014), "Virtue Epistemology and Epistemic Twin Earth", European Journal of Philosophy 22: 335-357.

Pritchard, D. (2014), “The Modal Account of Luck”, Metaphilosophy 45: 594-619.

(2015) “Anti-Luck Epistemology and the Gettier Problem”, Philosophical Studies 172: 93-111.

Rysiew, P. (2011), "Epistemic Contextualism", en E. Zalta, ed., The Stanford Encyclopedia of Philosophy. URL $=<\mathrm{http} / /$ plato.stanford.edu/archives/ win2011/entries/contextualism-epistemology/>

Sainsbury, R. M. (1997), "Easy Possibilities”, Philosophy and Phenomenological Research 57: 907-919.

Sosa, E. (1999), "How to Defeat Opposition to Moore", Philosophical Perspectives 13: $141-154$. 